\title{
Dynamic Range Method using Hiddenmarkov Models in RSA and DSA Algorithm
}

\author{
Noor Mohd, Ankur Dumka, Vijay Kumar
}

\begin{abstract}
Dynamic range approach has become a highest level of research field from last some year. The initiative beyond this process researched field is the reality that some particularrange are used in current environment and also which is used or deploy in an in effectivemanner. Theoverall wireless various range are allocated and fixed, but it is not need to be used. So, at this time the requirement for research in various wireless technologies expanding and there is no space for the wireless various rangesto assignimportant frequency bandsrelated to the future wireless technologies. Therefore, this is a reason to increase the use of the various ranges. To target this, we must be calculating the method for sharing the range. Our purpose to find out the wireless sensors' techniques, artificial Intelligencetechniques, cloud computing techniques for updating verification time of dynamic range approach. So, we used in this paper the Hidden Markov Model for defining the various types of range characteristics and forecast therangesample that will be obtained in future related methods. This forecast is used to define the RSA and DSA in better its verification time. The method for utilization HMM technique for minimizing the verification time of RSA and DSA will be executed in NS2 and Matlab simulator
\end{abstract}

Index Terms--- Cognitive Radio, Dynamic Range Approach, Hidden Markov Model, RSA Algorithm, DSA Algorithm

\section{INTRODUCTION}

Intelligent wireless $[1,5,78,11$ and 22] is apromising various technologywhich can be used to enhance applicationfor efficiency of wireless range, by give the permissionto other user (OU) to coincide with the side of firstuser (FU) among the range allocating.A private or publickey need for other user is that $\mathrm{SU}$ communication will not be change the first user'sexecution.To reach this, analgorithmrequires of beforeobserving by other users ifat the minimum one first user is forward, i.e. indicate to as "range robservingstandard".If there is no message are observed, the other users are grant to communicate.Signal observation has been mostlyevaluating above the previous decades, inside useless contexts of demand, motivate by a small of those innovative works, a number of observationanalyses have been advised to observefirst usercommunication after there are several agreereceiver at the other users.Asystematicframework is to consider that nothing is sanctioned at the secondary user receiver, such that, no sound and channel information are familiar.So, for this, the dummyalert andobservationpossibilityhave been examined after there is one primary user signal.So, as per given example the following orders are for example, in following arrangements whereas structural multiplexing algorithm are engagedor in other hand primary users communicates concurrently.
Moreover, the number of primary user signals is to be surelyforecast to generate as authentication by the predict use of huge antenna arrays and by the tendencyrespected to the extraloadand heterogeneous networks.As a result, we continually conflictframework whereas the number of receiver antennas is greater than the primary user signals.

\section{CHALLENGES IN ARTIFICIAL INTELLIGENT RADIO}

There are severaltechnical issues of Intelligent Radio which are defined below [3]:

\section{Type of Hardware Related Requirements}

Range recognize for intelligent wireless radios rangefind out for artificial intelligent wireless radios wants increase the rate of data transfer, and analog and digital type converters beside the side of very high vibrant capacity, and increase the speed signal processors. There are various soundanalysis methods had used for most successful receiving node diagram, as various type of network elimination, data modeling and for expandhandoffs, manipulationrules, and network allocation technologies.

\section{Problem Obtain Hidden theMain User}

In agent sense variousexamine, invisible main user issue and nodeissue. Further,motivate through too many methodsattachinghardtwo or more path vanish or shadowed noticed by prohibited user as observing for main user's presence. Figure-2 displays visual to the hidden user problemsand other two cercle indicate shows the working whereas the two circles displays working opportunity for main user and the prohibited wireless methods. Artificial intelligent wireless mechanism cause interference in the main user because the main communication'ssignal might no more observed due to invisibleplaces of nodes.

\section{Observing the Range Spectrum for Primary Users in FHSS} and DSSS

Two types of technology are used for securedtechniques, permanent frequency and range and also two type of range technologies are used:

- Frequency Hopping Spectrum and [2 and 3]

- Direct Sequence Range Spectrum [5 and 6].

FHSS mechanisms wide range of frequency band and its divided in various channels for communication one channel to another in order between the transmitter and receiver. Therefore, the DSSS (Direct-Sequence Spread Spectrum)methods are comparable to FHSS methods, though, both uses for the reduce the overall signal interference. 


\section{Frequency Range and Duration of WirelessSensing}

Frequency can be declaring by authorized key users at any time as prohibited user is working on their various range. If interference has to be ended to and start main authorized key holder, prohibited wireless concern to be intelligent to focus the incident of main users as rapidly as predictable and also concern to quite the spectrum instantly. That is why, observing technology concern to be able to identify the circumstances of main users inside summery of time duration. This condition verifies a correct on the presentation of observing algorithm and creates a trial for artificial intelligent wireless sensing design.

\section{VARIOUS ISSUESOF SPECTRUM SHORTAGE}

The whole spectrum usually fully allocated, and leave no free space for new wireless services and thesepolicies of current spectrum make it difficult for fast provide of new wireless services, which are most important in the case of emergency services like natural disaster etc.Further,most of the know that the spectrum frequency is never fully utilised temporally and spatially, because of which the the large portion created on the spectrum, which are known as white spaces or spectrum holes.

And the demand for wireless spectrum is increasing continuously because of growing need for wiresconnectivity. There are increasing number of users every year and along with their need of wireless services.But federal agencies and emergency services had placed high and uncompromising constraints on the spectrum. The current regulatory constraints do not allow the flexibility of dynamic spectrum reuse even when the licensed spectrum is perfect.

This rigid spectrum thus the main cause for the spectrum scarcity problem in future. So, there is a need of elevation in advanced applications with higher data rates according to the phenomenal advancements in wireless telecommunication method that is why have to take the radio spectrum as a scarce resource. In spite of the truth revealed by the administrative studies that radio spectrum is highly underutilized in both spatial and temporal domains. This underutilization places a tough challenge in front of governmental and national regulating bodies, who are responsible for regulating the spectrum constraints to satisfy the increasing demand for wireless connectivity with the help of future technology like wireless sensor, artificial intelligent technology etc.

Several communication commissions issued the spectrum guidelinestask enforcementdata record in 2002 with the goal of efficient, intelligent and clear use of this various resource.This data recordapplied the substitutions of today rigid guidelines with the new changeable spectrum guidelines according to dynamic spectrum allotment.This RSA and DSA algorithmprovide a very highreason for research in the artificial intelligent area, by allowing prohibited user approachThis thesis introduces the RSA and DSA algorithm and presents the RSA and DSA methodologies in framework-based networks system.

\section{THE SOLUTION OF SPECTRUM SHORTAGEISSUES}

There has been already high research attempt for sufficientfrequency rangeused.New wireless service can be fit to novelusers that have been assigned to authorized key users who use the spectrum in bad manner.

In June 2002 FCC had commissioned the Spectrum Policy Task Force report for improving the spectrum rigid guidelines.

The report has suggested many methods to improve spectrum use,such as reuse of the spectrum, exploitation of the spectrum, and transformation of the current fix system into variableservice-based system environment.

\section{V.}

\section{APPLICATION OF INTELLIGENT RADIO}

There are various applications which are related to Intelligent Radio are defined below [4,5,9,10,12,15 and 17]:

\section{Smart Grid Network System}

To resolve the issues relating to energy independence and sustainability, global warming and emergency resilience challenges, many governments are promoting the transformational of the $20^{\text {th }}$ century power grid into smart grids. Smart grids are of three high level layers, the physical power layer, the communication networking layer and the application layer. Smart grid can transform the way power is generated,delivered,consumed and billed. By adding intelligence, we can improve grid reliability, demand handling and responsiveness, increase efficiency and integrates renewable/distributed energy sources and reduce costs for the providers and the consumers.

\section{Public Safety Networks System}

The excellent use of wireless communication is by the various emergency service providers like, natural disaster, police, war, fire and emergency medical services. Therefore, the higher data rates are needed, reliability and delay orders vary from task to tasks or system to system.

\section{Cellular Network System}

Cellular networks have been shows to be very high significant in current years with consumer's projections of being always linked anywhere and at any time means that the all service always on mode. Theinitiation of smart mobile phones, networkingwebsites like YouTube, what-sup of novelinstruments such as online shopping, e-readers, smart classes concept have all added to the usage of cellular wireless network system environment.

\section{Wireless Medical Networks System (MMNS)}

In current years there has been increase in hospitalized people admission for high signs like body temperature, blood pressure,blood level oxygenand electrocardiograph (ECG) ultrasound digital x-ray etc., These are focused by body sensors that are linkedby wires and make difficulty, thus allowing sensors that are connected wireless reliably so that doctors or medical related people can respond frequently as per their accordingly.

\section{THE MOTIVATION OF THE RESEARCH}

Dynamic spectrum access [13 and 14] has become a high motivation for the researcher's in the last currentyears. The only motive behind this research area is the fact that fixed spectrum we have but still utilized very ineffective. Thetotal spectrum is assigned to the authorized users but not necessarily being used.

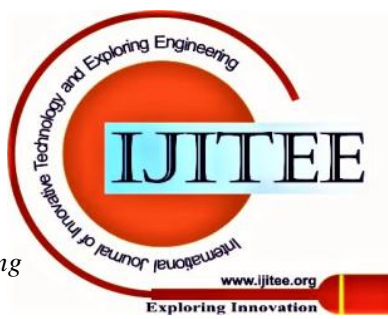


At the same time, theneed is growing frequently for the wireless sensor's services, since there are no chances of wireless spectrum for use the future wirelesssensor services as we have the fixrangeresources, so the only option is to use the existing one fully and efficiently. Tofulfilledthis, we must find the technologies to share the frequency spectrum distribution.

\section{DYNAMIC SPECTRUM ACCESS}

"Dynamic spectrum approach is a method that allow unauthorized user(secondary user) to access the spectrum of licensed user(primary user) without knowing of first user."

\section{Basic System}

Basic system refers to the guidelines system, by modulating bodies like FCC and the given system has all the advantages to approach the allocated spectrum range for the system.

\section{Other System}

Other type of systemstands to the without guidelines or intelligentsystem, and can be approach the spectrum only dynamic which is currently not used by the basic system.

\section{FUTURE PROPOSED WORK}

As per hidden markov model is a famous analytical tool for designing a wide range of time series datatype. The hidden markov model is alsoanalytical statistical method for designing producingseries of states. Markov model have found approach in various field and the given example in signal processing system, and in particular speech processingsystem [16,18,19,20 and 21].

Hidden markov model is a strong statistical analytical model in which the system being modelled is supposed to be a markov related process with noticed or hidden states of arrangements. In a systematic markov model the state is straight visible to the user side, and that is why the state of transition chances are the only variables. In a hidden markov model the output depends on the visible states but the state is not directly visible resources. Every state has a chances distribution over the achievable output tokens. This is how the order of output token by an HMM gives few information about the order of various states types.

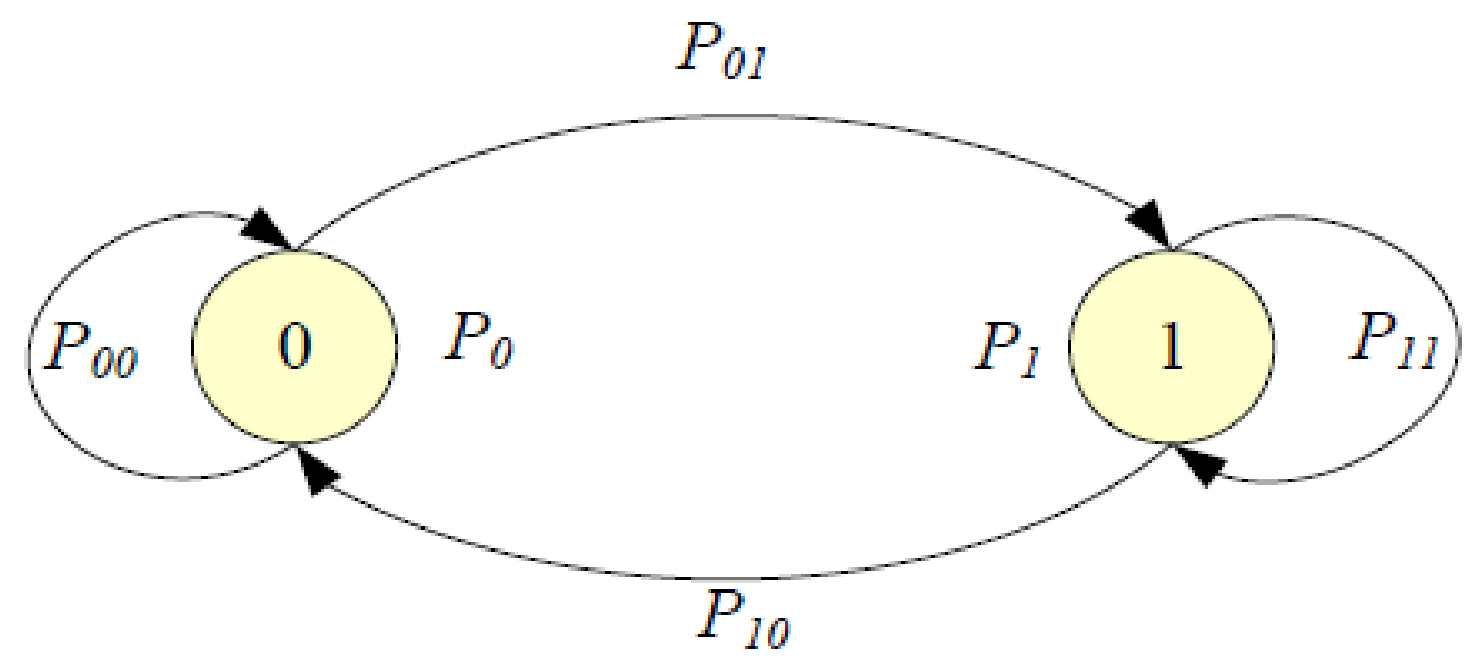

Figure 1: Simple Markov Model For Channel Occupancy States.

\section{THE RESEARCH APPROACH IN THIS AREA}

The taskspoint to use wireless communication technology and artificial intelligence methods Itgives to use Hidden Markov Model for sampling the spectrum nature and forecast the spectrum level that will be find out in future technologies. Thisforecast is used utilized to help the RSA and DSA in updating its verificationtime. Themethod for using HMM technique for decreasungverification time of RSA and DSA algorithm will be executed in NS2 and Matlab simulators. The methodextracts as its inputs data order upto given current time.Every data orderstand for a single snapshot of spectrum as a order of 1's and 0's. The spectrum is discretized in fixed sized of bands.A 0 indicate the band is free and 1 indicatethat the band already in use with a first user's signal communication.The method will forecast the sequence order that will be noticed at $t+1$. This is specified as input to the spectrum sensing amethod based on FFT (Fast Fourier transform) executed in NS2 and Matlab simulator.The spectrum sensing method will verify only those bands that have big probability of being free as evaluated by HMM algorithm. These bands are having 0's in sequence output by HMM algoritm at $\mathrm{t}+1$ time slot.

\section{DYNAMIC SPECTRUM APPROACH}

The RSA and DSA methods makes possible the effective using the radio spectrum by letting approach for unsubscribed other users to the free spaces or spectrum introducing in the radio spectrum[3-5].These free spaces or spectrum introducing are explain as region of the radio spectrum unused at a some time and location[4]. The problem in enabling the other userreceive develop from the interference with the first users, who can be showed because the reliable or authorized people in the spectrum band. TheRSA and DSA methods allows other user access to the quickly underutilized area of the spectrum so your access to the first users is going to be safe and protected and uninterrupted[2-5]. 


\section{Dynamic Range Method using Hiddenmarkov Models in RSA and DSA Algorithm}

This necessity of protecting the principal individual from interference, the other user terminals should have a determination creating capacity based on RSA and DSA algorithm guidelines. These wise other user terminals are named as artificial intelligent radio for the channel access.

XI. HIDDEN MARKOV MODEL METHOD
The figure-2 defined the working of HMM algorithm which is start from the multiple sequence alignment and then the insertions or deletions can be modelled and also after this process occupancy and frequency at each level in alignment are encoded and also define the flow chart in figure- 3 as Flow Chart for First and Other User to access the channel frequency range

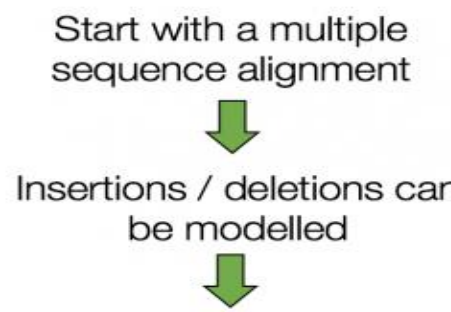

Occupancy and amino acid frequency at each position in the alignment are encoded

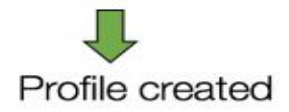

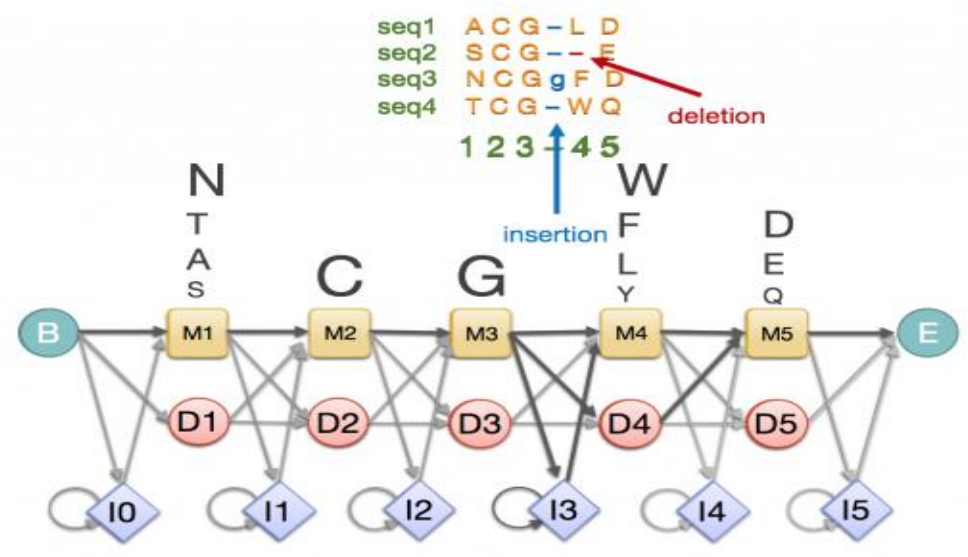

Figure-2 A profile HMM modelling a multiple sequence alignment

\section{Developed Algorithm for Decreasing VerificationTime}

1.Declare the spectrum band to some white space

2.Find out "HMM" possibility based on the current band

3.Use the "perfect band" possibility to evaluatethe requiredvarious frequencies

- Required number of transitions from state $\mathrm{n}$ (to state $\mathrm{m}$ )

- Required number of being in state $\mathrm{m}$ (and observing $O_{t}$ )
- Required number of starting in state $m$ 4.Sense the expected frequencies to estimate the parameters from observation state

$\left\{O_{t+1} \ldots \ldots \ldots \ldots . . . . . . t\right\}$

5.Decrease the band size by 1 at time instant $t+1$

6.If Band size reach to 1 Repeat 2-4 until the optimum band is not find that is no longer to use.

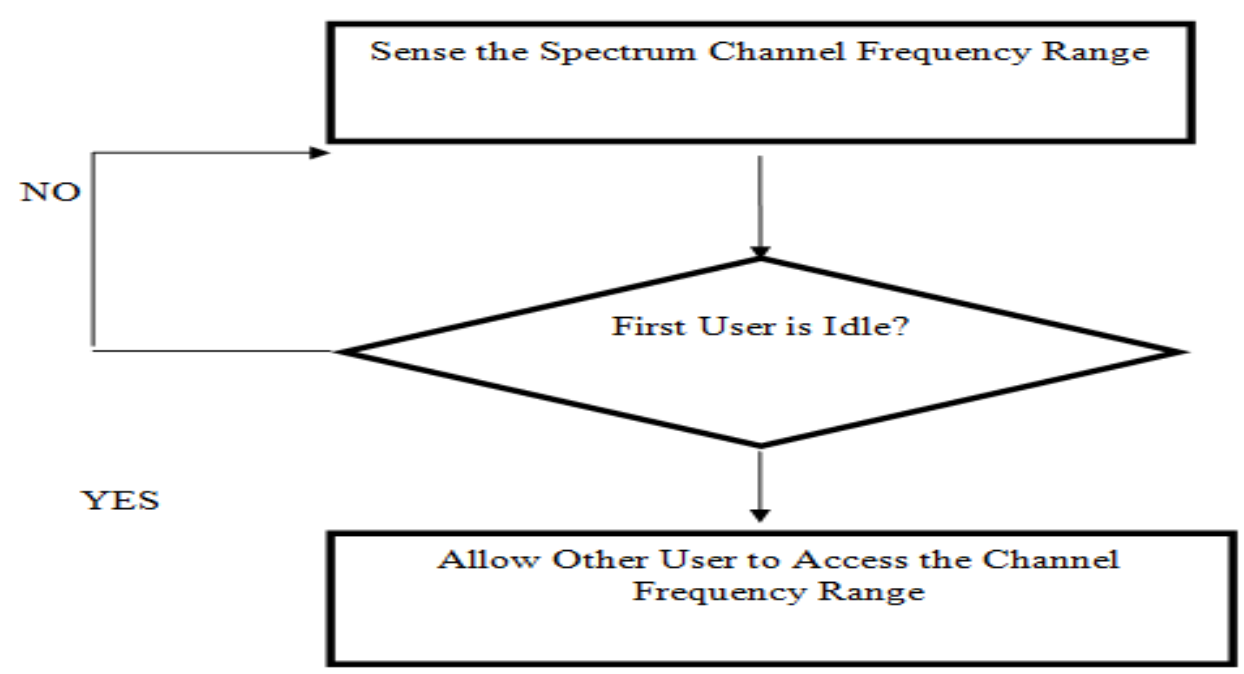

Figure 3: Flow Chart for First and Other User to access the channel frequency range

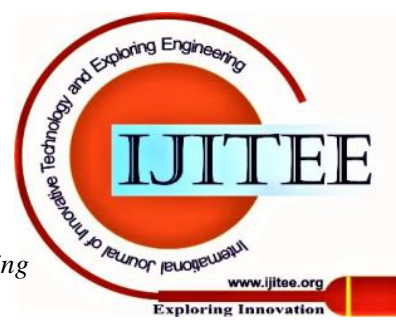


XII.

\section{FINAL RESULTS DISCUSSION}

The method for utilising HMM algorithm for decreasingverification time of RSA and DSA algorithm has been executed in NS2 amd Matlab simulator.The mathod takes as its inputs data ordere upto given current time.Every data orderindicate a single snapshot of spectrum as a range of 0 's and 1 's. The spectrum is discretized in predetermine sized bands.A 0 represents the band is free and 1 indicate that the band is already in use with a First User's signal.Thismethodidentify the range that will be noticed at $\mathrm{t}+1$. This is given as input to the spectrum sensing methoddepend on FFT (Fast Fourier transform) executed in various simulator. The spectrum sensing method will verify only those bands that have high large probability of being free as evaluated by HMM. These bands are having 0's in range output by $\mathrm{HMM}$ at $\mathrm{t}+1$.

As the bands now being calculated by Spectrum Sensing method are low in number the algorithm will complete the verification in low time. The verification results of spectrum sensing algorithm giving spectrum snapshots will become input to the next no.of iteration of HMM are used. The number of bands being verified spectrum sensing method will keep on reducing and when it completes a minimum count, the HMM is restarted with spectrum snapshot of complete band.So, some results in a sawtooth shaped plot of scan time of RSA and DSA algorithm with time as shown in (Figure 4-6).

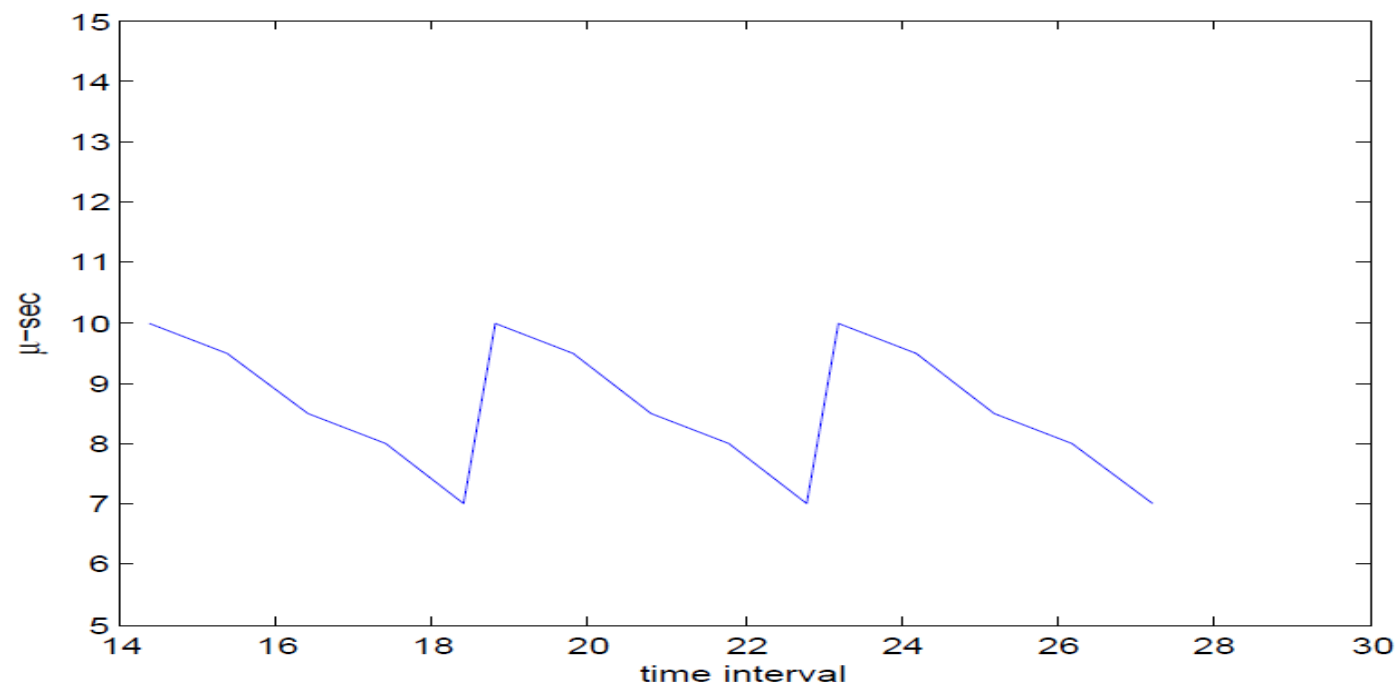

Figure 4: Various Time Interval with the respect to Search Time

Another plot shows the comprision between spectrum access without HMM and with HMM in term of number of free holes and scan time for searching a free holes in microseconds. This graph shows how the suggested HMM based RSA and DSA technique updates over the RSA and DSA technique without HMM.The scan time shown for HMM based RSA and DSA is the evaluated by taking the average of scan time values over at the timeduration.

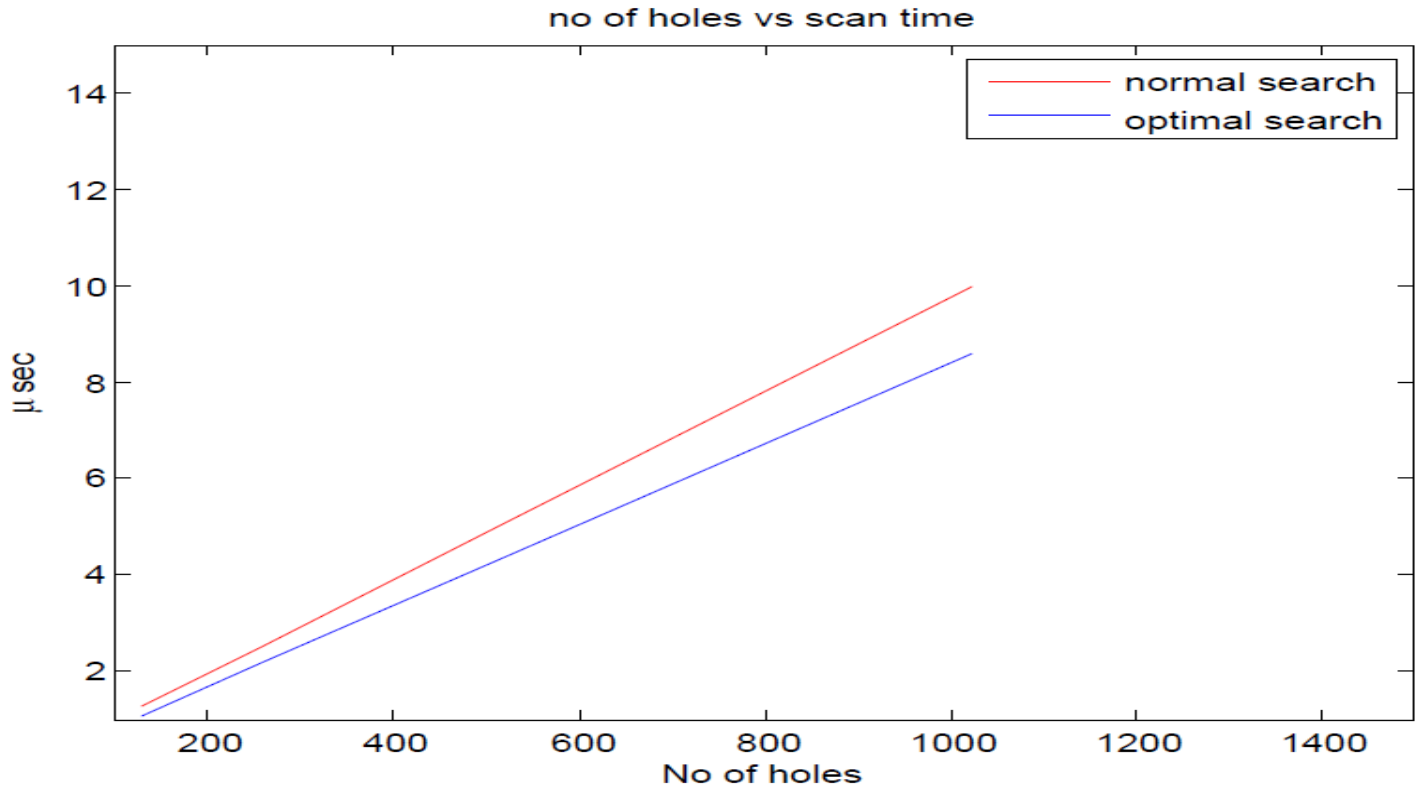

Figure 5: No. Of Holes with the respect to the Scan Time 


\section{Dynamic Range Method using Hiddenmarkov Models in RSA and DSA Algorithm}

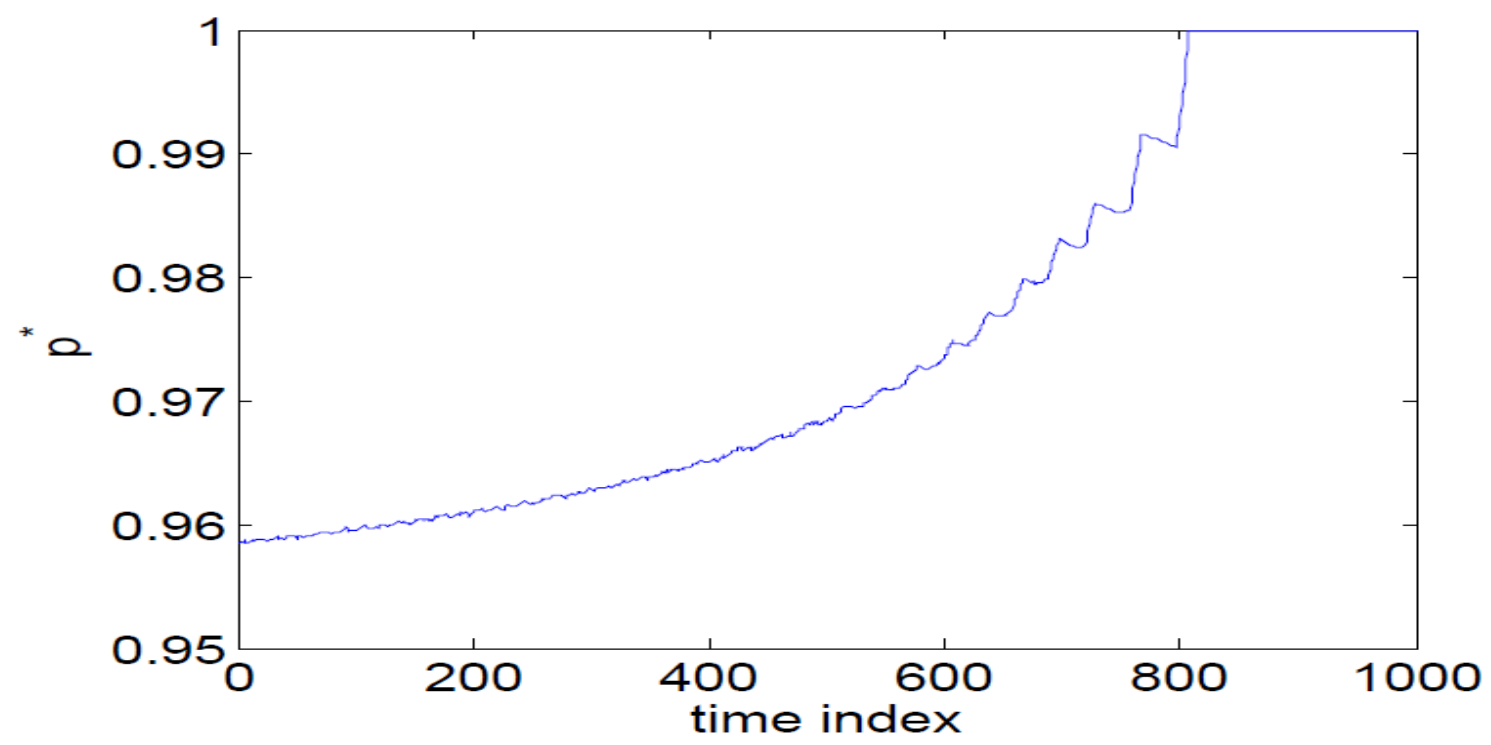

Figure 6: Time Index with the respect Probability

\section{CONCLUSION}

This paper proposed an approach based on HMM for decreasing the verification time of dynamic spectrum access in artificial intelligent radio.The simulation results show that the approach defined does improve the verification time of RSA and DSA algorithm.Also,this approach is limited in terms of the size of new snapshots of RSA and DSA algorithms that remains on decreasing as the algorithm progresses and requirements to start again from verifying the complete band.The work can be further extended by designing an approach removes this restriction and thus leading to more efficient use of spectrum in further communication or transmission.

\section{REFERENCES}

1. Morales-Jimenez, David, Raymond HY Louie, Matthew R. McKay, and Yang Chen. "Multiple-Antenna Signal Detection in Cognitive Radio Networks with Multiple Primary User Signals." arXiv preprint arXiv:1405.6408 (2014).

2. Liu, Yang, Zhangdui Zhong, Gongpu Wang, and Dan Hu. "Cyclostationary Detection Based Spectrum Sensing for Cognitive Radio Networks." Journal of Communications 10, no. 1 (2015).

3. Yucek, Tevfik, and Hüseyin Arslan. "A survey of spectrum sensing algorithms for cognitive radio applications." Communications Surveys \& Tutorials, IEEE 11, no. 1 (2009): 116-130.

4. Lu, Xiao, Ping Wang, Dusit Niyato, and Ekram Hossain. "Dynamic spectrum access in cognitive radio networks with RF energy harvesting." Wireless Communications, IEEE 21, no. 3 (2014): 102110.

5. Wang, Jianfeng, Monisha Ghosh, and Kiran Challapali. "Emerging cognitive radio applications: A survey." Communications Magazine, IEEE 49, no. 3 (2011): 74-81.

6. Bkassiny, M.; Yang Li; Jayaweera, S.K.,"A Survey on MachineLearning Techniques in Cognitive Radios",IEEE,Communications Surveys \& Tutorials, IEEE,2013

7. Salami, G.; Durowoju, O.; Attar, A.; Holland, O.; Tafazolli, R.; Aghvami, H.,"A Comparison Between the Centralized and Distributed Approaches for Spectrum Management",IEEE,Communications Surveys \& Tutorials, IEEE, 2011

8. Gronsund, P.; MacKenzie, R.; Lehne, P.H.; Briggs, K.; Grondalen, O.; Engelstad, P.E.; Tjelta, T.,"Towards spectrum microtrading",IEEE,Future Network \& Mobile Summit (FutureNetw), 2012,2012

9. Lili Cao; Haitao Zheng,"Distributed Rule-Regulated Spectrum Sharing",IEEE,Selected Areas in Communications, IEEE Journal on, 2008
10. Mangold, S.; Jarosch, A.; Monney, C.,"Operator Assisted Cognitive Radio and Dynamic Spectrum Assignment with Dual Beacons Detailed Evaluation",IEEE,Communication System Software and Middleware, 2006. Comsware 2006. First International Conference on, 2006

11. Wang Fan; Lu Fang; Geng Xuan,"Researching on future spectrum management based on cognitive radio",IEEE,Computer Application and System Modeling (ICCASM), 2010 International Conference on, 2010

12. Chung-Wei Wang; Li-Chun Wang,"Analysis of Reactive Spectrum Handoff in Cognitive Radio

13. Networks",IEEE,Selected Areas in Communications, IEEE Journal on, 2012

14. Axell, E.; Leus, G.; Larsson, E.G.; Poor, H.V.,"Spectrum Sensing for Cognitive Radio : State-of-the-Art and Recent Advances",IEEE,Signal Processing Magazine, IEEE,2012

15. Abadie, A.; Wijesekera, D.,"Leveraging an Inventory of the Cognitive Radio Attack Surface",IEEE,Cyber Security (CyberSecurity), 2012 International Conference on, 2012

16. Brown, T.X.; Jaroonvanichkul, S.,"Policy-based radios for UAS operations",IEEE,Globecom Workshops (GC Wkshps), 2012 IEEE, 2012

17. Wysocki, T.; Jamalipour, A.,"Pricing of Cognitive Radio Rights to Maintain the Risk-Reward of Primary User Spectrum Investment",IEEE,New Frontiers in Dynamic Spectrum, 2010 IEEE Symposium on,2010

18. Li-Chun Wang; Chung-Wei Wang; Adachi, F.,"Load-Balancing Spectrum Decision for Cognitive Radio Networks",IEEE,Selected Areas in Communications, IEEE Journal on,2011

19. Sheng-Yuan Tu; Kwang-Cheng Chen; Prasad, R.,"Spectrum Sensing of OFDMA Systems for Cognitive Radio Networks",IEEE,Vehicular Technology, IEEE Transactions on,2009

20. Peng Jia; Mai Vu; Tho Le-Ngoc; Seung-Chul Hong; Tarokh, Vahid,"Capacity- and Bayesian-Based Cognitive Sensing with Location Side Information",IEEE,Selected Areas in Communications, IEEE Journal on,2011

21. Lingjie Duan; Jianwei Huang; Biying Shou,"Investment and Pricing with Spectrum Uncertainty: A Cognitive Operator's Perspective",IEEE,Mobile Computing, IEEE Transactions on,2011

22. Wei Feng; Jiannong Cao; Chisheng Zhang; Liu, C.,"Joint Optimization of Spectrum Handoff Scheduling and Routing in Multihop Multi-radio Cognitive Networks",IEEE,Distributed Computing Systems, 2009. ICDCS '09. 29th IEEE International Conference on, 2009 .

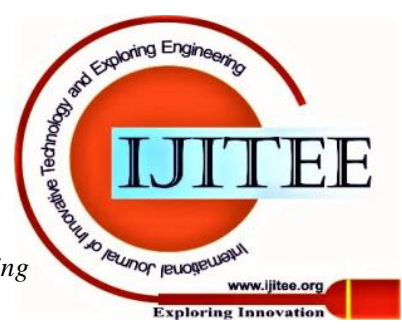

